\title{
Wave dynamic processes in cellular detonation reflection from wedges
}

\author{
Zongmin Hu • Zonglin Jiang
}

Received: 5 September 2005 / Revised: 16 August 2006 / Accepted: 4 September 2006 / Published online: 16 December 2006

(C) Springer-Verlag 2006

\begin{abstract}
When the cell width of the incident detonation wave (IDW) is comparable to or larger than the Mach stem height, self-similarity will fail during IDW reflection from a wedge surface. In this paper, the detonation reflection from wedges is investigated for the wave dynamic processes occurring in the wave front, including transverse shock motion and detonation cell variations behind the Mach stem. A detailed reaction model is implemented to simulate two-dimensional cellular detonations in stoichiometric mixtures of $\mathrm{H}_{2} / \mathrm{O}_{2}$ diluted by Argon. The numerical results show that the transverse waves, which cross the triple point trajectory of Mach reflection, travel along the Mach stem and reflect back from the wedge surface, control the size of the cells in the region swept by the Mach stem. It is the energy carried by these transverse waves that sustains the triple-wave-collision with a higher frequency within the over-driven Mach stem. In some cases, local wave dynamic processes and wave structures play a dominant role in determining the pattern of cellular record, leading to the fact that the cellular patterns after the Mach stem exhibit some peculiar modes.
\end{abstract}

Keywords Cellular detonation - Wedge - Reflection . Wave dynamics $\cdot$ Simulation

The English text was polished by Yunming Chen.

Z. Hu $(\varangle) \cdot$ Z. Jiang

LHD, Institute of Mechanics, CAS, Beijing 100080, China

e-mail: bighumin@126.com

Z. Hu

ReCAPT, Gyeongsang National University, Jinju,

Kyeongnam 660-701, South Korea

\section{Introduction}

Recently an increasing number of investigations have been carried out on detonation reflections from wedges. Some questions still remain unanswered, such as the influence of the reflection on the detonation wave front structures, the wave dynamic phenomena occurring in the reflection processes, and the interaction between the inside-cell transverse waves and the main transverse wave of Mach reflection and their effect on the trajectory of triple point collision. It is well known that in a detonation front structure, the leading shock is wrinkled, consisting of alternate weak incident shocks and stronger Mach stems, and jointed at the triple points by transverse waves which travel back and forth perpendicular to the wave front and extend back into the reaction zones. The transverse waves interact with the reaction zones and shear layers behind the leading shock, and induce endothermic or exothermic combination depending on the local wave structures. When an incident detonation wave (IDW) diffracts over a wedge, such unsteady and nonequilibrium processes normally cause complicated wave phenomena of interest. Moreover, when the cell width of IDW is comparable to or larger than the Mach stem height, self-similarity will fail during IDW reflection over wedges.

Zhang et al. [1] reported a large scale experiments on detonation reflections in acetylene-air mixtures. The transition from a regular to Mach reflection was found to take place in the Mach reflection regime predicted by the three-shock theory . Guo et al. [2] and Thomas et al. [3] experimentally visualized the detonation reflection from wedges and smaller detonation cells behind the Mach stem were demonstrated by a smoked foil record technology. For Mach-reflected detonations, it 
was found that the main trajectory triple points are always not in a straight line $[2,4,5]$. However, both the present numerical simulations and cellular patterns from experiments are not informative enough to interpret the curved trajectory due to the low resolution. Moreover, in most of pioneering researches [4,6,7], the idealized single-step or two-step reaction models were applied to simplify the chemical reaction kinetics which involves actually multiple species and multiple characteristic reaction scales. Nevertheless, it is known that, for detonation simulations, quantitatively different chemical kinetics can produce quantitatively different results. Hu et al. [8] simulated various cases of detonation reflections over wedges with a detailed chemical reaction model and demonstrated difference in the trajectory of triple points between shock waves in inert gases and non-cellular detonations in combustible mixtures of hydrogen and oxygen.

In this paper, the cellular detonation reflection from wedges is investigated for the wave dynamic processes occurring in the wave front, including transverse shock motion and detonation cell variations behind the Mach stem. A detailed reaction model is implemented to simulate two-dimensional (2D) cellular detonations in stoichiometric mixtures of $\mathrm{H}_{2} / \mathrm{O}_{2} / \mathrm{Ar}$.

\section{Governing equations}

With usual assumption that dissipative effects, such as viscous, heat-conductive and diffusive effects, are neglected, the governing equations of gaseous detonations are the 2D multi-component Euler equations with chemical reaction source terms. The equations can be written in conservation form with ns continuity equations for a perfect gaseous mixture in Cartesian coordinates:

$\frac{\partial \boldsymbol{U}}{\partial t}+\frac{\partial \boldsymbol{F}}{\partial x}+\frac{\partial \boldsymbol{G}}{\partial y}=\boldsymbol{S}$,

where,

$$
\boldsymbol{U}=\left[\begin{array}{l}
\rho C_{1} \\
\rho C_{2} \\
\vdots \\
\vdots \\
\rho C_{n s} \\
m \\
n \\
E
\end{array}\right], \quad \boldsymbol{F}=\left[\begin{array}{l}
m C_{1} \\
m C_{2} \\
\vdots \\
\vdots \\
m C_{n s} \\
m^{2} / \rho+p \\
m n / \rho \\
(E+p) m / \rho
\end{array}\right]
$$

$$
\boldsymbol{G}=\left[\begin{array}{l}
n C_{1} \\
n C_{2} \\
\vdots \\
\vdots \\
n C_{n s} \\
m n / \rho \\
n^{2} / \rho+p \\
(E+p) n / \rho
\end{array}\right], \quad \boldsymbol{S}=\left[\begin{array}{l}
\dot{\omega}_{1} \\
\dot{\omega}_{2} \\
\vdots \\
\vdots \\
\dot{\omega}_{n s} \\
0 \\
0 \\
0
\end{array}\right]
$$

where, $\boldsymbol{U}$ denotes unknown variables, $\boldsymbol{F}$ and $\boldsymbol{G}$ the fluxes, $\boldsymbol{S}$ the chemical reaction source term. $c_{i}=\rho_{i} / \rho(i=1, n s)$ is mass fraction of the species $i . m=\rho u, n=\rho v, u$ and $v$ are velocity components in $x$ - and $y$-direction, respectively. The density of each species is denoted by $\rho_{i}$. The total energy per volume $E$ is defined as

$E=\rho h-p+\rho\left(u^{2}+v^{2}\right) / 2$.

Each species is usually assumed to be a thermally perfect gas, and the specific heat and enthalpy for each species can be calculated by thermal polynomial equations as given in Ref. [9]. Then, the mixture enthalpy $h$ is the summation of all the partial enthalpies of each component as following:

$h=\sum_{i=1}^{n s} c_{i} h_{i}$.

According to Dalton's law, pressure $p$ is the sum of the partial pressures of all species, and can be calculated by the equation of state for a perfect gas.

$p=\sum_{i=1}^{n s} \rho_{i} R_{i} T$,

where, $R_{i}$ is the gas constant of species $i$, and $T$ is the temperature of the gas mixture. For an elementary chemical reaction, the chemical production rate $\dot{\omega}_{i}$, derived from a reaction mechanism of $n r$ chemical reactions, can be calculated by

$\dot{\omega}_{i}=W_{i} \sum_{r=1}^{n r}\left(v_{i r}^{\prime}-v_{i r}^{\prime \prime}\right)\left(k_{f r} \prod_{j=1}^{n s}\left(\chi_{j}\right)^{v_{i r}^{\prime}}-k_{b r} \prod_{j=1}^{n s}\left(\chi_{j}\right)^{v_{i r}^{\prime \prime}}\right)$,

where, $i$ and $j$ denote the species number in the $r$ th elementary reaction. The molecular weight of each species is denoted by $W_{i} . v_{i r}^{\prime}$ and $v_{i r}^{\prime \prime}$ are the stoichiometric coefficient of species $i$ in the $r$ th reaction appearing as the reactant and product, respectively. The molecular concentration of each species is denoted by $\chi_{j} . k_{f r}$ and $k_{b r}$ denote the forward and the backward reaction rates. The forward reaction rate of each reaction is calculated by the Arrhenius law [Eq. (8)] and the corresponding 
Fig. 1 Verification by comparison of numerical (lower half) and experimental Schlieren [3] (upper half) of detonations reflection over wedges with angle $\mathbf{a} 30^{\circ}, \mathbf{b} 60^{\circ}$ in conditions of $2 \mathrm{H}_{2}+\mathrm{O}_{2}+\mathrm{Ar}$, $P_{0}=20 \mathrm{kPa}$ at room temperature and mesh size of $\Delta=0.2 \mathrm{~mm}$
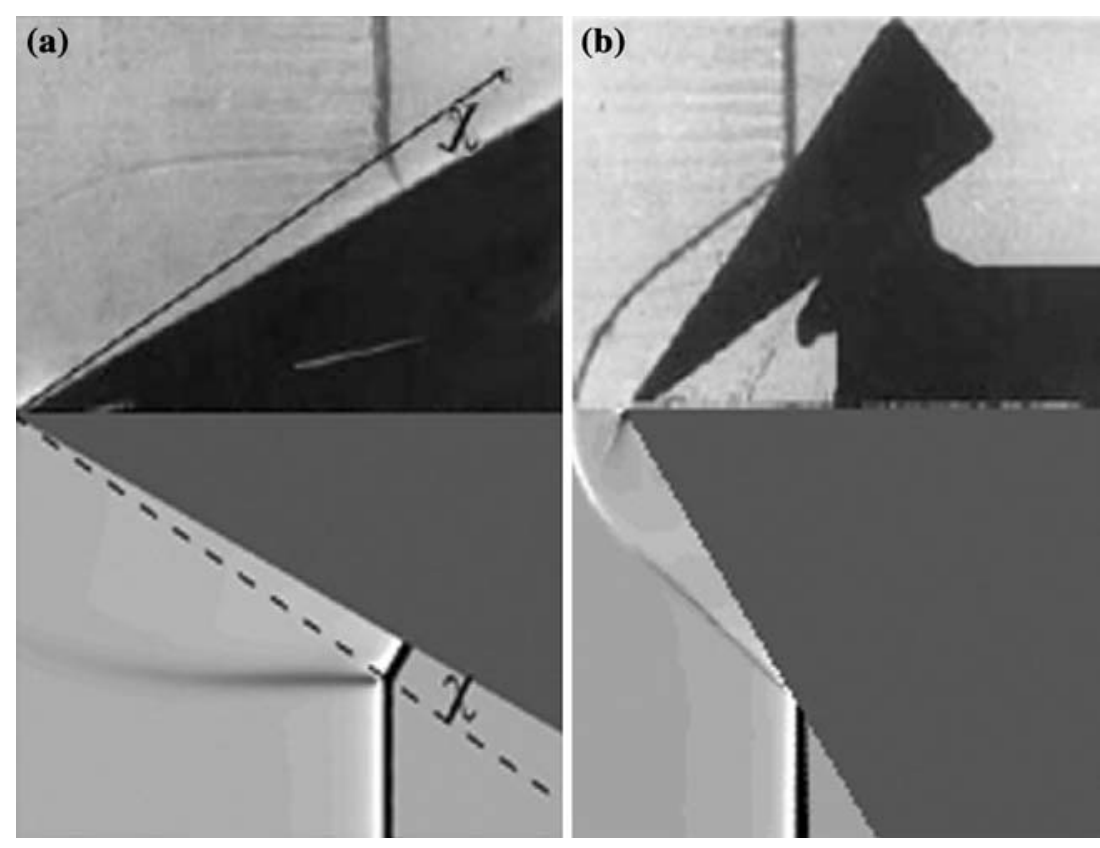

backward reaction rate can be derived from the equilibrium constant [Eqs. (9)-(11)] and $k_{f r}$.

$k_{f r}=C_{r} T^{n r} \exp \left[-E a_{r} / R T\right]$,

$\kappa_{b r}=\kappa_{f r} / \kappa_{e r}$,

$\kappa_{e r}=\kappa_{p r}\left(\frac{P_{a t m}}{R T}\right)^{\sum_{i=1}^{n s}\left(v_{i r}^{\prime \prime}-v_{i r}^{\prime}\right)}$,

$\kappa_{p r}=\exp \left(\sum_{i=1}^{n s}\left[\left(v_{i r}^{\prime \prime}-v_{i r}^{\prime}\right)\left(\frac{s_{i r}^{0}}{R_{i r}}-\frac{h_{i r}}{R_{i r} T}\right)\right]\right)$.

In the present study, the reacting species include $\mathrm{H}_{2}, \mathrm{O}_{2}$, $\mathrm{H}, \mathrm{O}, \mathrm{OH}, \mathrm{H}_{2} \mathrm{O}$, and a certain percentage of Argon as an inert diluent.

\section{Numerical algorithms}

Equation (1) is discretized in space by using the secondorder explicit dispersion-controlled dissipative (DCD) scheme [10,11] proposed by Jiang et al. in 1995 for shock wave capturing. The time marching integration is performed by using a Runge-Kutta algorithm with second-order accuracy. During the computation, the contribution from fluid dynamic terms is calculated first to obtain an intermediate value of $\tilde{\boldsymbol{U}}^{\prime}$, which is followed by a calculation accounting for chemical reaction contribution to evaluate $\tilde{\boldsymbol{U}}$ for the next time step. The approach allows separately solving fluid dynamic and chemical reaction contributions with different time steps to make the $\Delta t$ consistent with the CFL condition and the required time scale for the stiff ODES of the reactions.

\section{Numerical results and discussion}

\subsection{Verification}

Although cellular detonations involve slip layers which are unstable to the Kelvin-Helmholtz instability and jets which produce Rayleigh-Taylor-type instabilities, only macro phenomena in wave dynamics occurring in the reflection of gaseous detonations over wedges are of interest in this paper. To verify the numerical algorithms, detonation wave reflections from wedges with different wedge angles in stoichiometric mixtures of hydrogen and oxygen diluted by $25 \%$ Argon $\left(2 \mathrm{H}_{2}+\mathrm{O}_{2}+\mathrm{Ar}\right.$, $P_{0}=20 \mathrm{kPa}$ at room temperature) are simulated and then compared with experimental Schlieren. Two kinds of reflection mechanism of detonation waves, regular and Mach reflection are shown in Fig. 1, where the experimental pictures are put in the upper half and the numerical results in the lower half. From these figures, it can be seen that the angle of triple point trajectory, $\chi$ in Fig. 1a and the reflection angle in Fig. $1 \mathrm{~b}$ are in good agreement with the experiments.

\subsection{Chemical reaction scales and detonation cells}

The characteristic size of detonation cells and the degree of regularity depend on the composition of the fuel and initial thermal conditions. But the characteristic size 
usually is one or two orders of magnitude greater than the $1 \mathrm{D}$ steady detonation reaction length which is controlled by the non-dimensionalized activation energy $\hat{E} a=E a / R T$ of the detonatable mixtures [12]. Numerical simulations show that increasing $\hat{E} a$ favors more irregular cellular structures characterized by the higher frequency of appearance and disappearance of the triple points, larger variation of local shock speeds inside detonation cells, stronger shock wave interaction at triple points and deeper penetration of un-reacted gas pockets [7]. In nature, each elementary reaction has its own $\hat{E} a$ and the characteristic reaction length scale, which result in different effects on the cell size.

Figure $2 \mathrm{a}, \mathrm{b}$ show $1 \mathrm{D}$ variations of mass concentrations after the wave front of a ZND detonation and a cellular detonation, respectively. As shown in Fig. 2a, between the Champaman-Jouguet plane and the leading shock where $u_{C J}+a_{C J}=D=2198 \mathrm{~m} / \mathrm{s}$ and $p_{C J} / p_{0}=$ 16.7 according to $\mathrm{C}-\mathrm{J}$ theory for the considered reactive mixture, $\mathrm{H}_{2}$ decreases quickly to its equilibrium value due to the chain reactions, while the active radical $\mathrm{H}$ arrives its peak value shortly after the leading shock and then decreases nearly to its equilibrium state. However, $\mathrm{OH}$ arises slower and peaks in the region before the $\mathrm{C}-\mathrm{J}$ plane. Obviously, these species undergo reactions of different length scales. In the case of cellular detonation shown in Fig. 2b, radical $\mathrm{H}$ demonstrates a characteristic length of about $8 \mathrm{~mm}$ nearly similar to that in ZND detonation. At the same time, the reaction performance of $\mathrm{OH}$ is quite different from that in ZND case. It experiences vibrations in the propagation direction and maintains a comparative higher average concentration during a comparative longer distance. Furthermore, $\mathrm{OH}$ accumulates to a peak value immediately when the front arrives at point 2 as a result of triple point collision whereas it delays at the center of the cell, point 1 . Transverse wave collision, triple wave evolution, temperature variation and induction reaction in the flow field behind the cellular detonation front can answer for these differences.

Note that for the quantitative purpose, numerically generated cellular detonation patterns are superior to experimental ones because of the difficulty in depositing a uniform coating of soot on a foil in practical applications. To demonstrate the quantitative feature of the cell structure, numerical simulations have been proved to be more informative [13]. Initiated by the $1 \mathrm{D}$ wave front shown in Fig. 2a and transverse perturbations along the leading shock, 2D cellular detonation comes into being and evolutes to a stable pattern, as shown in Fig. 3 . Compared with results in the pioneering research which applied a simplified single step reaction model [7,12], our results are similar to their cases of lower activity (a)

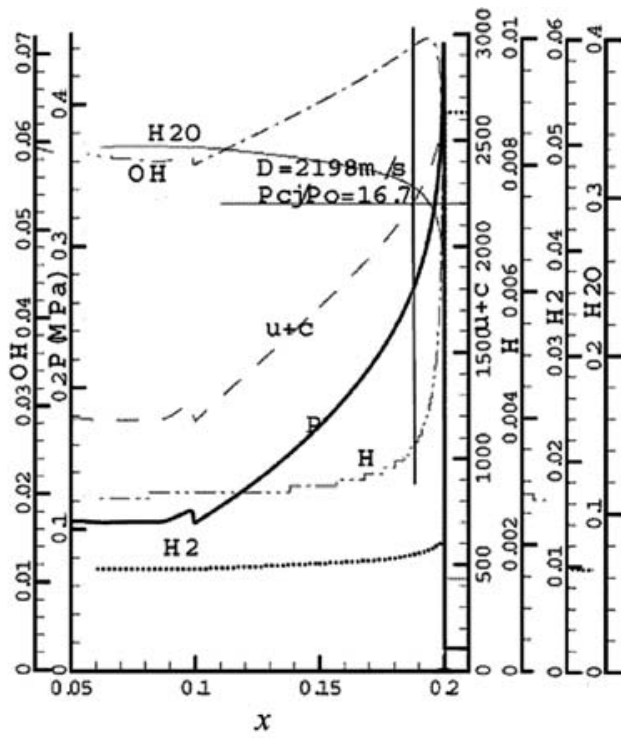

(b)

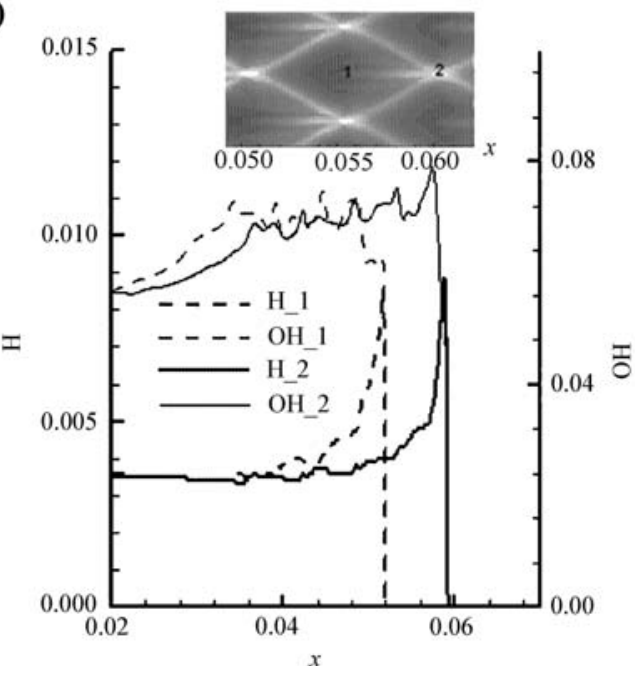

Fig. 2 a One-dimensional $(1 D)$ detonation wave and the distribution of mass concentration of species (detonation mixture: $2 \mathrm{H}_{2}+$ $\left.\mathrm{O}_{2}+\mathrm{Ar}, P_{0}=16 \mathrm{kPa}, T_{0}=298 \mathrm{~K}\right)$. b Two-dimensional cellular detonation and distributions of species concentration along the cell centerline (detonation mixture: $2 \mathrm{H}_{2}+\mathrm{O}_{2}+\mathrm{Ar}, P_{0}=16 \mathrm{kPa}$, $\left.T_{0}=298 \mathrm{~K}\right)$ at two time instants: the leading shock arriving at point 1 (dash line) and point 2 (solid line)

energy of $\hat{E} a=2.1$. But for detonation waves, quantitatively different chemical kinetics can produce quantitatively different results. In this study with a detailed elementary chemical model, it covers a longer way to stabilize the cellular variation from initial perturbations. It is reasonable that there are more characteristic reaction length scales affecting the cell generation and evolution process in the present case than the pioneering simulation in which only one chemical reaction length scale works. 
Fig. 3 Time-integrated maximum pressure contours of $2 \mathrm{D}$ cellular detonation showing cell pattern evolution, mesh size of $\Delta=0.083 \mathrm{~mm}$
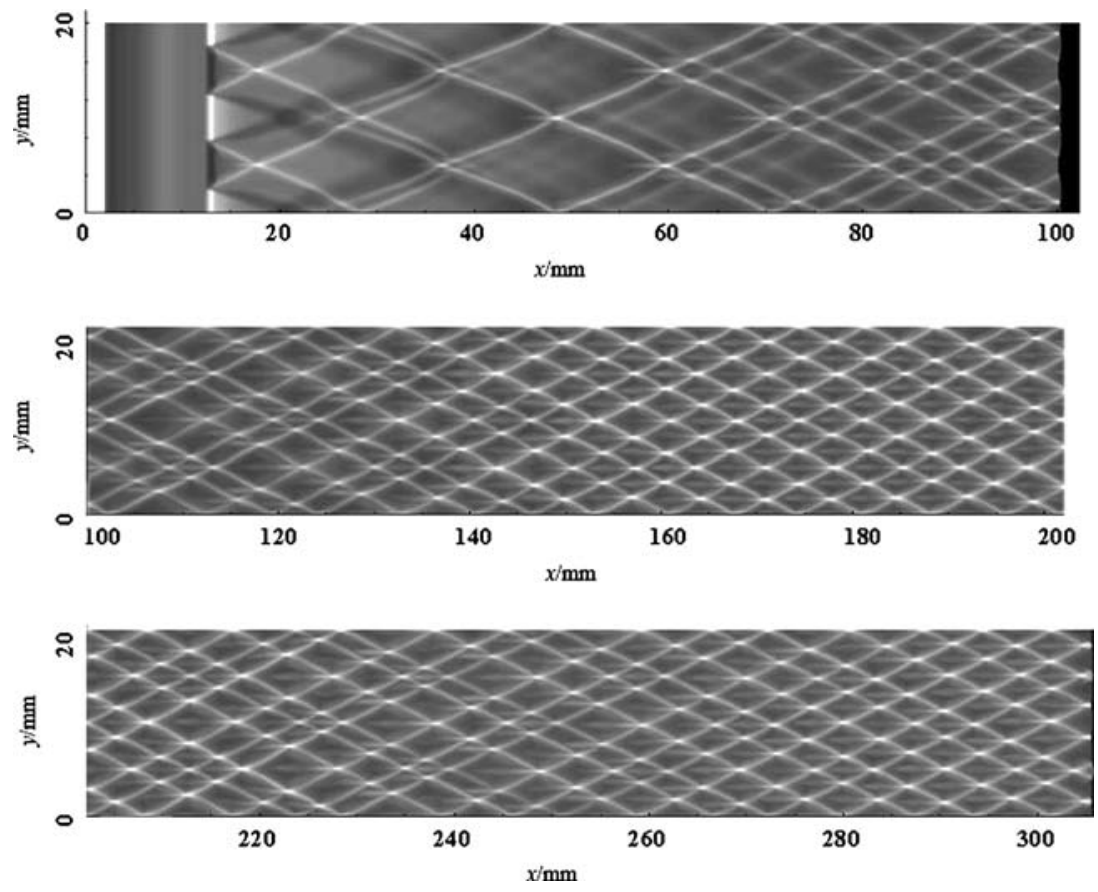

\subsection{Wave dynamics of Mach reflection}

One transient multi-wave structure of the detonation front is shown in Fig. 4 on the background of numerical cellular record. Like the diffraction of a nominally planar gaseous detonation over a wedge, the leading front of cellular detonation is divided into two parts, the cellular incident detonation wave is named as W-IDW (for convenience and same hereinafter) and the main or macro Mach stem is named as W-MS which is actually an overdriven detonation. These two parts and the main transverse wave of the macro reflection are named as $\mathrm{W}$-TW joint at the main triple points. The triple points form a trajectory named as W-Trajectory. Each triplewave structure unit along the wrinkled W-IDW or W-MS consists of a local incident shock, Mach stem and transverse waves, named as C-IDW, C-MS and C-TW, respectively. It is known that the C-TW propagates back and forth perpendicular to the wave front and joints the C-IDW and C-MS at the triple points which finally integrate the trajectory inside a cell named as C-Trajectory. The detailed structures are labeled in Fig. 4. The simulations are carried out by using a detonatable gaseous mixture of $2 \mathrm{H}_{2}+\mathrm{O}_{2}+\mathrm{Ar}$ at an initial pressure of $p_{0}=16 \mathrm{kPa}$ and room temperature.

As for the fact that the cell size itself keeps changing during Mach reflection along the wedge, no general conclusion can be drawn to make it clear. There is some evidence that the changes are due to higher temperature after the overdriven detonation wave which forms the

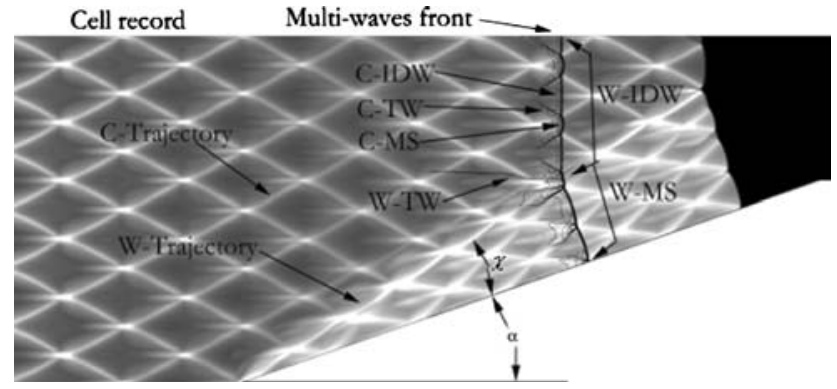

Fig. 4 Multi-wave detonation front reflecting from a wedge of angle $\alpha=19.3^{\circ}$, gas mixture of $2 \mathrm{H}_{2}+\mathrm{O}_{2}+$ Ar at initial pressure of $P_{0}=16 \mathrm{kPa}$ and $T_{0}=298 \mathrm{~K}$, and mesh size of $\Delta=0.083 \mathrm{~mm}$. (Note: W-Trajectory: main triple-point trajectory of detonation reflection; W-TW: main transverse wave or main reflected wave of detonation reflection; W-IDW: main incident detonation wave; W-MS: main Mach stem of detonation reflection; C-Trajectory: local triple-point trajectory inside a cell; C-IDW: local incident detonation wave inside a cell; $\mathrm{C}$-TW: local transverse waves inside a cell; C-MS: local Mach stem inside a cell. In above terminology, $\mathrm{W}$ - and $\mathrm{C}$ - means wave structure involves the macro reflection over the wedge and wave structure inside one detonation cell, respectively.)

Mach stem, and to lessen extent pressure effects. One must also consider that these transverse waves, C-TW, propagate laterally across the main reflected shock wave [3]. In some cases, local wave dynamic processes and fine structures may play a dominant role on the cell record along the wedge surface. As a result, the cell patterns after the Mach stem would exhibit some peculiar features. 
Figure 5 shows several successive instantaneous profiles of pressure along the centerline (marked by dotdot-dash line in the subfigure) of three conjoint cells (labeled as Cell-1, Cell-2, and Cell-3). Four pressure peaks appear due to the collisions of macro and local waves within these three cells. Cell-1 is entirely out of the region swept by the W-MS, while Cell- 2 and Cell3 lie on each side of the W-Trajectory. In Cell-1, the shock pressure decays quickly from its maximum value to a lower level towards the cell center point, and then rises to the next peak near the cell end. During the second half of the above process, the collision at the tails of two C-TWs (which form a structure like the comet tails following the cell end) drives up the shock pressures, but it never exceeds the maximum value at the collision point of the heads of corresponding transverse waves along the detonation wave front. In Cell-2, some quantitatively different things occur. After the similar decay process, the shock pressure climbs to a higher peak value rapidly and sharply due to the collision between the local C-TW and the global W-TW. Moreover, the impact between the tails of the two kinds of transverse waves mentioned above is so strong as to overrun the pressure of the corresponding leading shock and form a stronger comet wake structure. In Cell-3, as a result of the collision of stronger transverse waves in the region swept by the W-MS, the peak value of shock becomes higher than that in Cell-1, but still lower than that in Cell-2.

The evolution of the cellular front along the wedge is given in Fig. 6 by the instantaneous pressure contours according to the time sequence. In these figures, white parts represent high pressure values. The label above each subfigure means the sequence number of the corresponding cellular front, for example, F-385 is the 385th

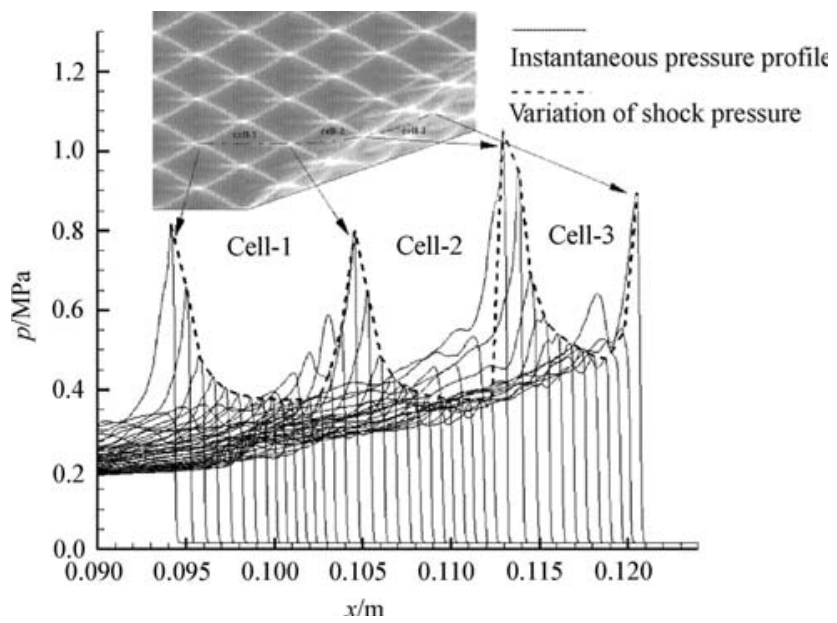

Fig. 5 Variations of pressure along the center lines of three cells near the wedge apex, the cell pattern is intercepted from Fig. 4 wave front. From F-385 to F-393, two C-TWs run face to face along the front and finally collide in the unreflected region. In this case, the first transverse wave impinges the wall a little before the wedge apex, as shown in F-390. Then from F-390 to F-403, it can be found that the reflected C-TW and the W-TW propagate synchronously and incorporate into one. Thus in Fig. 5 one can see that the W-Trajectory and the local C-Trajectory superpose each other. It is imaginable that if the wedge apex does not lie very close to the point where the local C-TW hits the wall, difference will occur, which will be presented hereinafter. From F-403 to F-415, the second triple point leaves the W-IDW, crosses the W-TW, moves along the W-MS, and then impacts the wedge surface. From subfigures such as F-403, F-415 and F-429, one can find that the W-TW decays due to the energy consumption in induction reaction of the mixture in the zone after the local incident shock wave. Consequently, it is reenhanced by the collision between the local triple point and the macro triple point. Subfigures from F-478 to F-500 show the development of transverse waves along the W-MS during a later stage. Due to smaller influence from the unsteady collision and communication between waves on both sides of the main triple point, they appear more regular. Consequently, the cells in this region are relatively uniform and regular as shown in Fig. 4. This is contrary to what mentioned in Ref. [5], in which smaller spacing of transverse waves was found at the foot of W-MS, while the transverse wave spacing behind W-IDW persisted in the region behind the W-MS. The reason may be that different scale, $L / \lambda$, is used in the present study, where $L$ denotes the length scale of the wedge and $\lambda$ the length scale of the cells. In these subfigures, local transverse waves in the region swept by the W-MS are stronger and extend farther into products than those in the un-reflected region.

As mentioned above, when the W-MS connects to a local incident shock, a C-IDW within the W-IDW, the W-TW then is attenuated because energy is transferred to the induction pocket (see subfigure F-415 in Fig. 6). In such phases, the local portion of the W-Trajectory bends towards the wedge surface consequently, such as the third cell boundary along the $\mathrm{W}$-Trajectory as shown in Fig. 4. In order to explain these unsteady and chemical non-equilibrium phenomena, mass fraction of $\mathrm{OH}$ and $\mathrm{H}$, temperature and pressure distribution flood are shown in Fig.7. In such lower temperature region behind the $\mathrm{C}$-IDW, more fraction of $\mathrm{H}$ and less of $\mathrm{OH}$ have been generated. This indicates that the chemical reaction of mixtures in this region is in the stage of induction, not the exothermic stage. As the reflected shock is re-enhanced shortly after the collision of the local triple point and the main triple point, the $\mathrm{W}$-Trajectory will be adjusted 
Fig. 6 Instantaneous pressure contour during cellular detonation reflection from a wedge of angle $\alpha=19.3^{\circ}$ for a gas mixture of $2 \mathrm{H}_{2}+\mathrm{O}_{2}+\mathrm{Ar}$ at conditions of $P_{0}=16 \mathrm{kPa}, T_{0}=298 \mathrm{~K}$, and a mesh size of $\Delta=0.083 \mathrm{~mm}$
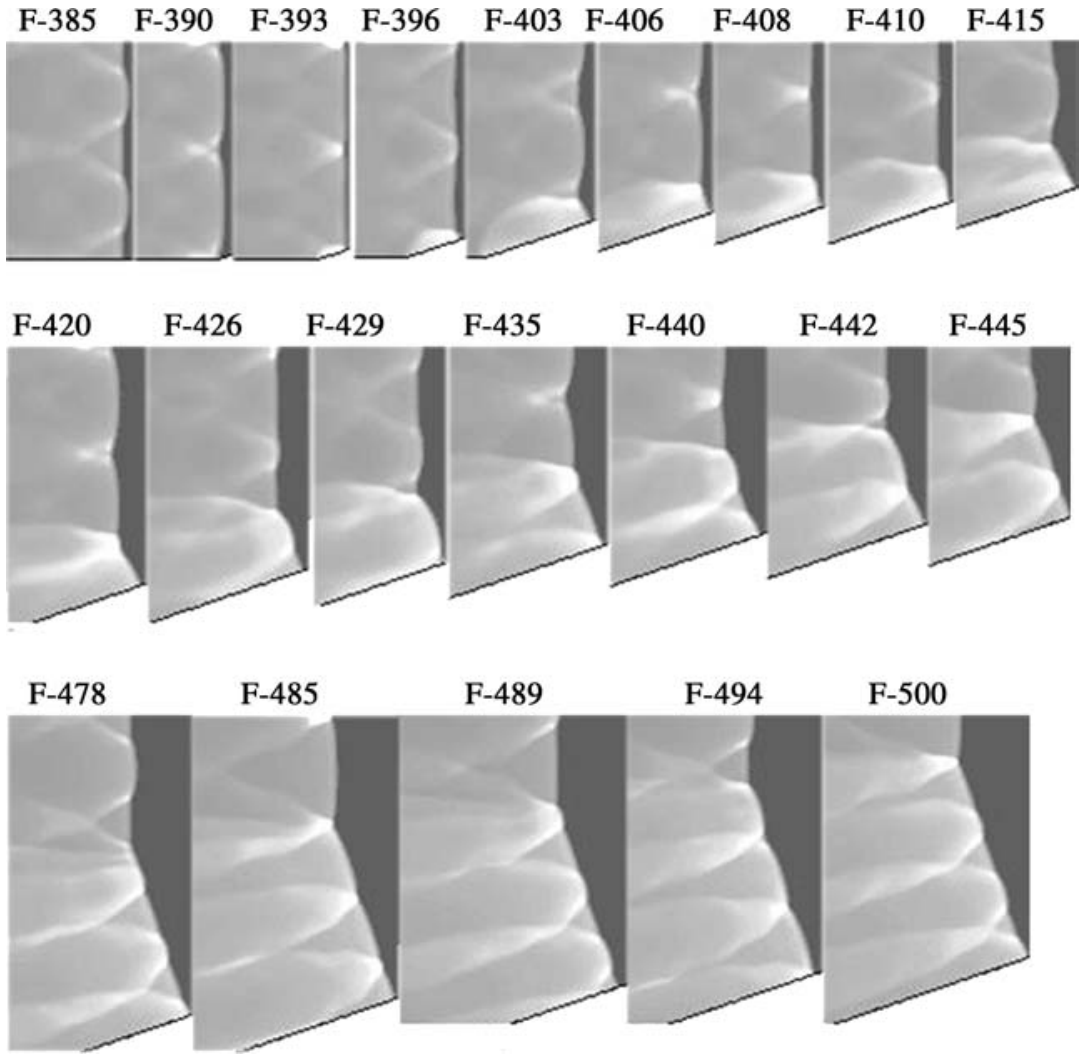

towards the original direction. In Fig. $7 \mathrm{~b}$, the induction zone shown by the distribution of $\mathrm{H}$ behind C-MS within $\mathrm{W}$-IDW is evidently longer than that behind the W-MS, but shorter than that behind the C-IDW above it, which is consistent with the thermodynamic parameters and chemical reaction rates in these regions. These different length scales can be seen in Fig. 7e quantitatively demonstrated by the length scale of radical $\mathrm{H}$. The local high temperature area in Fig. $7 \mathrm{c}$ and the domain of high $\mathrm{OH}$ concentration in Fig. 7a along the W-TW behind the induction pocket mentioned above make it clear that the exothermic reaction is strengthened by the collision between C-TW and W-TW. This process may be called as local reignition. Several exothermic islands scatter in the backward flowfield behind the leading front, as shown in Fig. 7a, which indicates that the reactive flow in this region is chemical non-equilibrium and nonuniform. Higher temperature regions can be found in Fig. 7c, which correspond to these exothermic islands.

\subsection{Triple-point trajectory of Mach reflection}

A numerical and an experimental soot record are integrated in Fig. 8. Due to C-TW reflecting back from the wedge surface, those cells near the W-Trajectory are split into smaller ones. Cells near the W-Trajectory both in the numerical (upper half) and experimental (lower half) cellular patterns appear irregular. Although the cell pattern is highly sensitive to the boundary condition, noises and perturbations within the actual flowfield, the cell structures appear determinate to certain extent. For example, the evolutions and patterns of cells near point 1 and 2 in the numerical results look very like those in the experiment record as shown in Fig. 8.

In the W-IDW region, the transverse wave changes its propagation direction twice. First it turns an angle $a$ (the wedge angle, see Fig. 4) when crossing the W-trajectory, and then impinges back when reflecting over the wedge surface. This is one of the main reasons that can explain the difference of cell size after the W-IDW and after the W-MS, beside the increase of thermodynamics parameters after the W-MS.

As shown by the numerical cell patterns in Figs. 4 and 8 , the wedge apex happens to lie at the vertex of one cell before the wedge apex. The trajectory of triple point of Mach reflection, W-Trajectory, looks like a straight line and just starts from the wedge apex. In Fig. 9, the wedge apex is adjusted and located at the center point of the nearest cell. The trajectory appears to be a curve and does not start from the wedge apex. The wave fronts at 
(a)

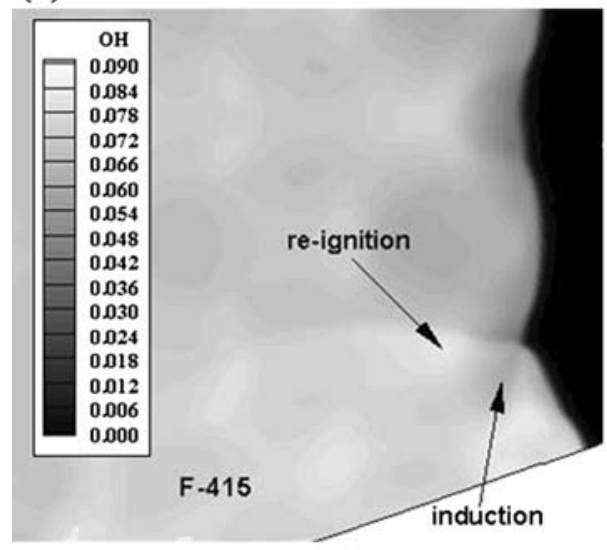

(c)

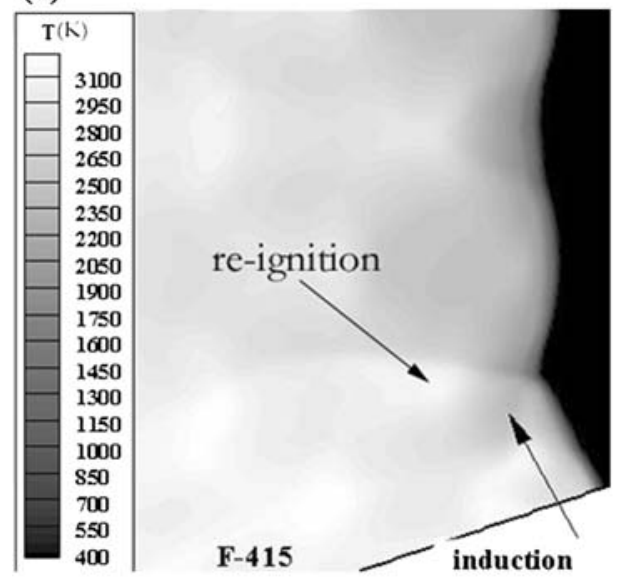

(b)

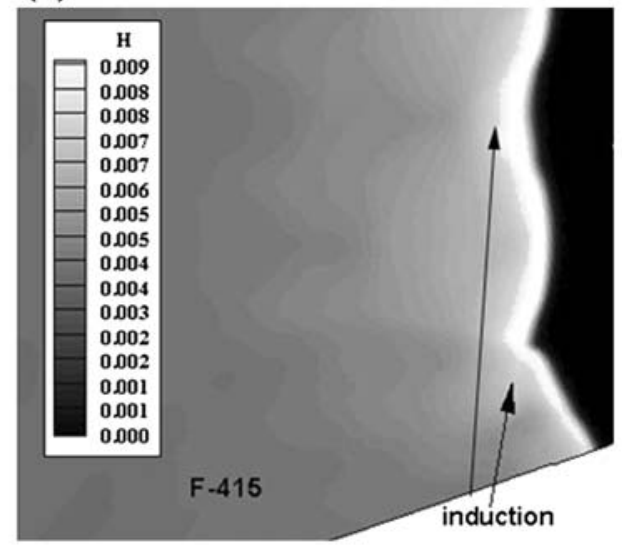

(d)

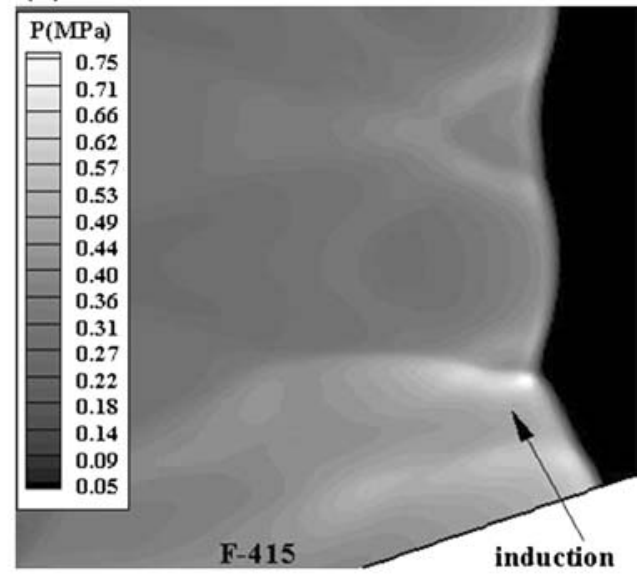

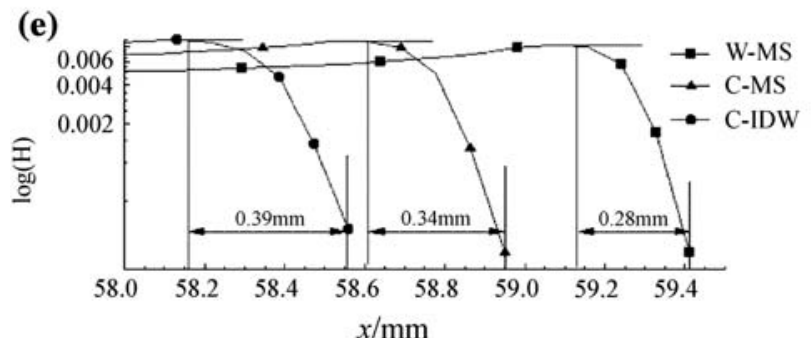

Fig. 7 Front structure (F-415) near the main triple point and state parameters: a Mass fraction of OH, b mass fraction of $\mathrm{H}$, c temperature, d pressure, $\mathbf{e}$ induction reaction length scales behind different waves shown by the mass fraction of $\mathrm{H}$

different time sequence are also shown by variation of pressure defined as follows:

$f(p)=\left((\partial p / \partial x)^{2}+(\partial p / \partial y)^{2}\right)^{0.5}$.

As to the start point of the trajectory, front F3 shows that it is still near the wedge apex. Because the cell boundaries record the historical peak value of pressure, it is the high pressure generated by the cell-inside transverse wave impinging on the wedge surface that overwrites the pressure of the triple point of macro reflection and makes the main trajectory invisible. Furthermore, as soon as the W-IDW begin to diffract form the wedge, its foot part happens to be the local C-IDW, which is weaker than C-MS. Consequently, the main triple point and the reflected wave are weak at the beginning, which makes the main trajectory even more difficult to be identified.

From Figs. 8 and 9, it can be seen clearly that the total number of the transverse waves is decreasing along the triple point trajectory of Mach reflection. For example, in the visual field of the upper half of Fig. 8, transverse waves incorporate three times in case that C-TWs and the W-TW propagate in the same direction. Hereinto, one occurs at the wedge apex, the other two occur near cells marked by 1 and 2 . It can be seen that, from wave 


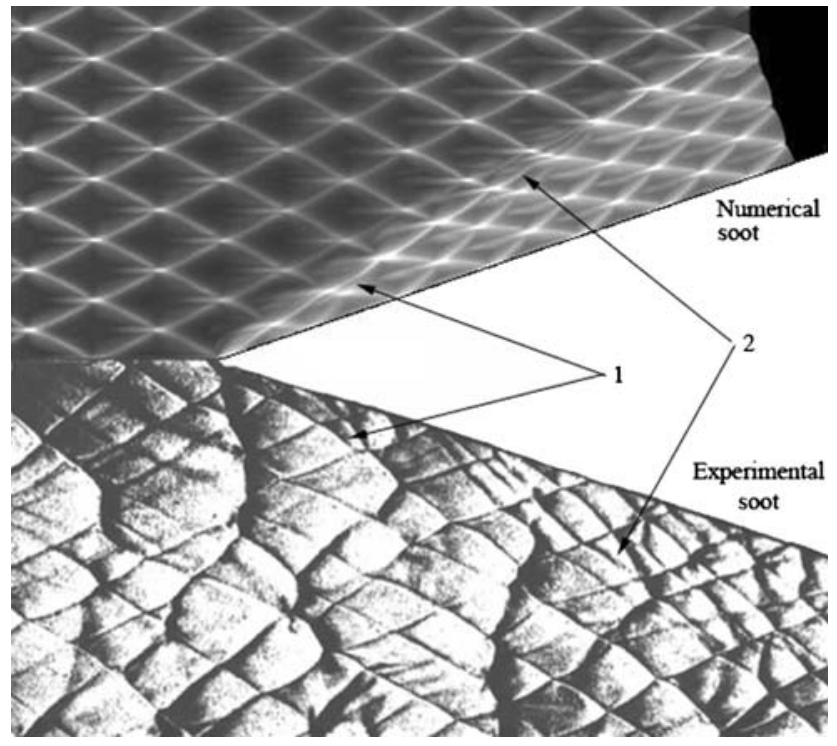

Fig. 8 Numerical (upper half) and experimental (lower half) soot in the Mach reflection region

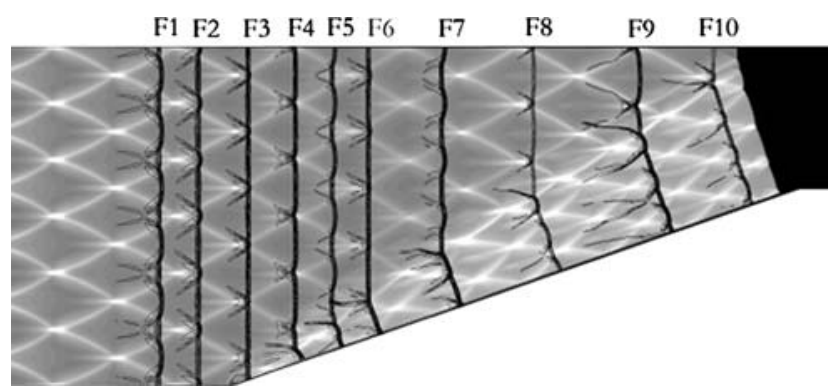

Fig. 9 Time-sequential cellular detonation wave fronts with time intervals between two consecutive wave fronts being $10 \Delta t, 13 \Delta t$, $12 \Delta t, 10 \Delta t, 8 \Delta t, 20 \Delta t, 26 \Delta t, 30 \Delta t, 26 \Delta t$, fronts shown by pressure variation

fronts F3 to F4 and from F7 to F8 in Fig. 9, these complicated processes are also obvious.

\section{Conclusions}

The process of 2D cellular detonations reflecting from wedges is numerically modeled on the basis of multispecies reactive Euler equations with an elementary reaction model. It can be concluded from the simulations that: (1) The C-TWs, which cross the W-Trajectory, travel along the W-MS and reflect back from the wedge surface, control the size of the cells in the region swept by the Mach stem, W-MS. (2) It is the energy carried by local transverse waves that sustains the triple-wavecollision with a higher frequency within the over-driven
Mach stem. (3) If the W-MS connects to a C-IDW within the W-IDW, the W-TW then will be attenuated due to energy transfer to the induction pocket, resulting in the fact that the local portion of the W-Trajectory bends towards the wedge surface. (4) The total number of transverse waves within the front decreases when the cellular detonation wave diffracts over a wedge. (5) The high pressure generated by the cell-inside transverse waves impinging on the wedge surface overwrites the pressure near the triple point of Mach reflection and makes the main triple-point trajectory invisible, which can explain why the trajectory is not a straight line and may not start from the wedge apex in some cases.

Acknowledgments This work was supported partially by the Korea Research Foundation Grant funded by the Korea Government (MOEHRD) (KRF-2005-005-J09901).

\section{References}

1. Zhang, F., Murray, S.B., Thibault, P.A.: Transition from regular to Mach reflection of detonation waves in acetylene-air mixture, pp. 339-341. In: Houwing A. F. P. (ed.) Proceedings of the ISSW21. Springer, Berlin Heidelberg New York (1997)

2. Guo, C.M., Zhang, D.L., Xie, W.: The Mach reflection of a detonation based on soot track measurements. Combust. Flame 127, 2051-2058 (2001)

3. Thomas, G.O., Williams, R.L.: Detonation interaction with wedges and bends. Shock Waves 11, 481-492 (2002)

4. Ohyagi, S., Obara, T., Nakata, F., Hoshi, S.: A numerical simulation of reflection processes of a detonation wave on a wedge. Shock Waves 10, 185-190 (2000)

5. Akbar, R.: Mach reflection of gaseous detonations. Ph.D. Dissertation, Rensselaer Polytechnic Institute, Troy (1997)

6. Sharpe, G.J.: Transverse waves in numerical simulations of cellular detonations. J. Fluid Mech. 447, 31-51 (2001)

7. Gamezo, V.N., Desbordes, D.: Formation and evolution of two-dimensional cellular detonations. Combust. Flame 116, 154-165 (1999)

8. Hu, Z.M., Gao, Y.L., Zhang, D.L., Yang, G.W., Jiang, Z.L.: Numerical simulation of gaseous detonation reflection over wedges with a detailed chemical reaction model (in Chinese). Acta Mech. Sin. 36(4), 385-391 (2004)

9. Mcbride, B.J., Zehe, M.J., Gordon, S.: NASA Glenn coefficients for calculating thermodynamic properties of individual species. NASA/TP 2002-211556, Glenn Research Center, Cleveland (2002)

10. Jiang, Z., Takayama, K., Chen, Y.S.: Dispersion conditions for non-oscillatory shock capturing schemes and its applications. Comp. Fluid Dyn. J. 4, 137-150 (1995)

11. Jiang Z.L.: On dispersion-controlled principles for non-oscillatory shock-capturing schemes. Acta Mech. Sin. 20(1), 1-15 (2005)

12. Lee, J.H.S.: Dynamic parameters of gaseous detonations. Annu. Rev. Fluid Mech. 16, 311-336 (1984)

13. Lee, J.H.S.: Detonation waves in gaseous explosives. In: Ben-Dor, G., Igra, O., Elperin T. (eds.) Handbook of Shock Waves. Academic, New York (2001) 